\title{
Surface motion of a semi-elliptical hill for incident plane SH waves*
}

\author{
Jianwen Liang* and Jia Fu \\ Department of Civil Engineering, Tianjin University, Tianjin 300072, China
}

\begin{abstract}
A closed-form analytical solution of surface motion of a semi-elliptical cylindrical hill for incident plane SH waves is presented. Although some previous analytical work had already dealt with hill topography of semi-circular and shallow circular, our work aims at calculating surface motion of very prolate hill for high incident frequency, and explaining the special vibrating properties of very prolate hill. Accuracy of the solution is checked by boundary conditions, numerical results for surface motion of oblate and prolate hills are calculated, and some conclusions are obtained.
\end{abstract}

Key words: semi-elliptical hill; analytical solution; plane SH wave; surface motion; oblate hill; prolate hill; Mathieu function; high frequency

CLC number: P315.3

Document code: A

\section{Introduction}

One of the major concerns of strong-motion seismology is to explain and quantify seismic characteristics of local topographies. It may be studied either by analytical or numerical methods. The analytical method is often called wave function expansion method. Numerical methods are methods such as the finite differences, finite elements, boundary elements, etc. The advantage of numerical methods is that they can be applied to local topographies of arbitrary shape, while analytical methods are essential for exploring the physical nature and checking the accuracy of numerical methods.

The pioneering work for the analytical solutions was done by Trifunac $(1971,1973)$, and he developed the closed-form analytical solutions of surface motion of a semi-cylindrical alluvial valley or canyon for incident plane SH waves, then the solutions were extended to the case of a semi-elliptical alluvial valley or canyon by Wong and Trifunac (1974a, b), and to the case of a shallow circular alluvial valley and canyon by Yuan and Liao (1994, 1995).

However, the closed-form analytical solutions of

\footnotetext{
* Received 9 August 2011; accepted in revised form 22 September 2011; published 10 October 2011.

† Corresponding author. e-mail: liang@tju.edu.cn

(c) The Seismological Society of China and Springer-Verlag Berlin Heidelberg 2011
}

surface motion of a semi-cylindrical or a shallow circular hill for incident plane SH waves were developed more recently by Yuan and Men (1992) and Yuan and Liao (1996) using auxiliary function method. Then the solutions were improved to higher incident frequency by sine and cosine series expansion technique (Lee et al., 2006).

Compared with a semi-cylindrical hill or a shallow circular hill, a semi-elliptical hill is more approximate for natural convex topography, and especially it can simulate a very prolate hill.

This paper presents a closed-form solution of surface motion of semi-elliptical hill for incident plane $\mathrm{SH}$ waves by using the sine and cosine series expansion technique proposed by Lee et al. (2006), and aims at giving results for some very prolate hills for high incident frequency and explaining some vibrating properties.

\section{The model}

As shown in Figure 1, the model to be analyzed in this paper is located in elliptical coordinates which define a family of confocal ellipses and confocal hyperbolas intersecting with each other orthogonally. We describe an oblate hill by $\left\{(\xi, \eta) \mid \xi=\xi_{0}, 0 \leq \eta \leq \pi\right\}$ with $y \leq 0$ for its half-space, and a prolate hill by $\left\{(\xi, \eta) \mid \xi=\xi_{0}\right.$, $-\pi \leq \eta \leq-\pi / 2 \bigcup \pi / 2 \leq \eta \leq \pi\}$ with $x \geq 0$ for its half-space. The material property of soil is described by its rigidity 


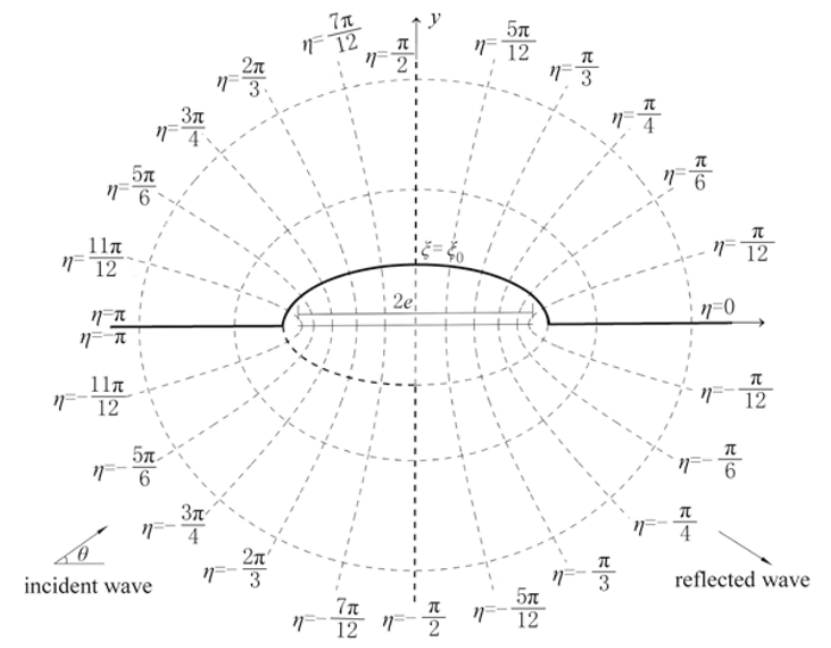

Figure 1 The model in elliptical coordinates. An oblate hill is defined by $\left\{(\xi, \eta) \mid \xi=\xi_{0}, 0 \leq \eta \leq \pi\right\}$ with $y \leq 0$ for its half-space, or a prolate hill is defined by $\left\{(\xi, \eta) \mid \xi=\xi_{0},-\pi \leq \eta \leq-\pi / 2 \bigcup \pi / 2 \leq \eta \leq \pi\right\}$ with $x \geq 0$ for its half-space. $\mu$ and shear wave velocity $\beta$. The half-space is elastic, isotropic and homogeneous.

\subsection{Elliptical coordinates}

Elliptical coordinates define a family of confocal ellipses and confocal hyperbolas intersecting with each other orthogonally. The relationship between Cartesian coordinates $x-y$ and elliptical coordinates is

$$
\left\{\begin{array}{ll}
x=e \cosh \xi \cos \eta & 0 \leq \xi<\infty \\
y=e \sinh \xi \sin \eta & 0 \leq \eta \leq 2 \pi
\end{array},\right.
$$

where $\xi$ is radial coordinate and $\eta$ is angular coordinate with $e$ being the focal length of ellipse. The minor-tomajor axial ratio $M$ is defined as

$$
M=\frac{\text { minor axis } B}{\text { major axis } A}=\tanh \xi .
$$

With different minor-to-major axial ratio $M$, we can define either oblate hills or prolate hills, and some typical oblate hills or prolate hills shown in Figure 2 are studied in this paper.

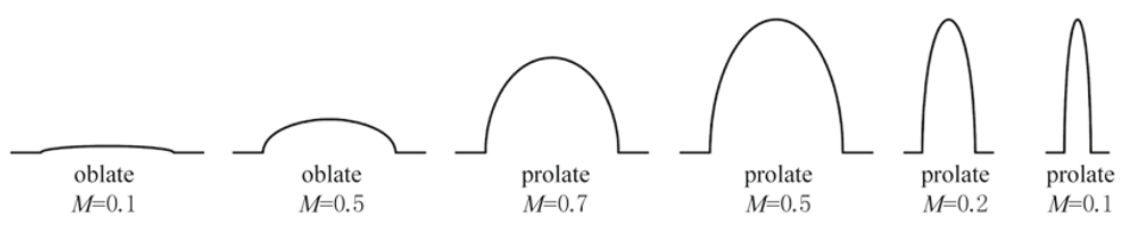

Figure 2 Hill with different minor-to-major axial ratios $M$.

The two-dimensional Helmholtz equation in elliptical coordinates for scalar displacement potentials $\varphi$ is

$$
\left(\frac{\partial^{2} \varphi}{\partial \xi^{2}}+\frac{\partial^{2} \varphi}{\partial \eta^{2}}\right)+e^{2} k^{2}\left(\cosh ^{2} \xi-\cos ^{2} \eta\right) \varphi=0
$$

where $k=\omega / \beta$ is wave number.

Separating variables by $\varphi(\xi, \eta)=X(\eta) Y(\xi)$ for equation (3), it comes two independent ordinary differential equations:

$$
\begin{gathered}
X^{\prime \prime}(\eta)+(b-2 q \cos 2 \eta) X(\eta)=0, \\
Y^{\prime \prime}(\xi)-(b-2 q \cosh 2 \xi) Y(\xi)=0,
\end{gathered}
$$

where $q=e^{2} k^{2} / 4$ and $b$ is the characteristic number. The solutions for equation (4a) are called angular Mathieu functions:

$$
\left\{\begin{array}{l}
c e_{2 m}(\eta, q)=\sum_{r=0}^{\infty} A_{2 r}^{2 m}(q) \cos 2 r \eta \\
s e_{2 m+2}(\eta, q)=\sum_{r=0}^{\infty} B_{2 r}^{2 m+2}(q) \sin 2 r \eta \\
c e_{2 m+1}(\eta, q)=\sum_{r=0}^{\infty} A_{2 r+1}^{2 m+1}(q) \cos (2 r+1) \eta \\
s e_{2 m+1}(\eta, q)=\sum_{r=0}^{\infty} B_{2 r+1}^{2 m+1}(q) \sin (2 r+1) \eta
\end{array}\right.
$$

There are several normalization conventions of angular Mathieu functions and here we adopt the following orthogonal series:

$$
\begin{gathered}
\int_{0}^{2 \pi} c e_{m}^{2}(\eta, q) \mathrm{d} \eta=\int_{0}^{2 \pi} s e_{m}^{2}(\eta, q) \mathrm{d} \eta= \\
\pi \text { (or other constant). }
\end{gathered}
$$

The solutions for equation (4b) are called radial Mathieu functions: 


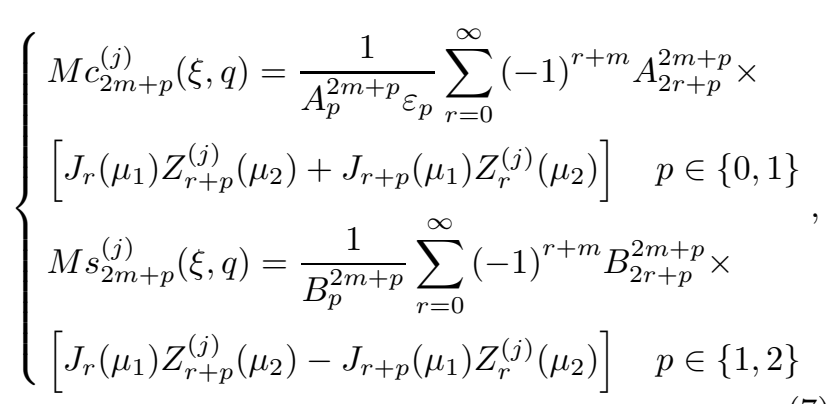

where $\varepsilon_{0}=2, \varepsilon_{1}=1, \mu_{1}=\sqrt{q} \exp (-\xi), \mu_{2}=\sqrt{q} \exp (\xi)$, and $Z^{(j)}(\cdot)$ means the Bessel function of $j$ th kind.

With $q \rightarrow 0$, the ellipse regresses into a circle and the confocal hyperbolas regress into radius of the circle. Also, angular Mathieu functions $c e_{m}(\eta, q)$ and $s e_{m}(\eta, q)$ become trigonometric functions $\cos m \eta$ and $\sin m \eta$ while radial Mathieu functions become Bessel functions.
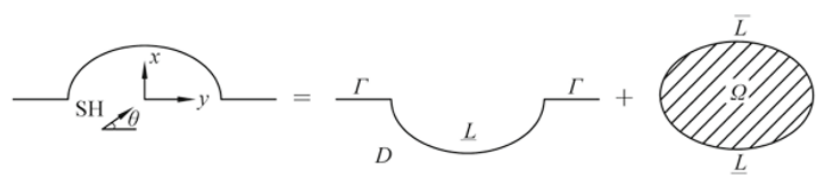

Figure 3 Two regions model.

\subsection{Free-field motion}

It is assumed that the incident plane SH wave $u$ has out-of-plane motion in $z$-direction with circular frequency $\omega$, incident angle $\theta$ and amplitude of 1 ,

$$
u=\exp \left[-\mathrm{i} \omega\left(t-\frac{x}{\beta} \cos \theta-\frac{y}{\beta} \sin \theta\right)\right] .
$$

The motion $u$ can be expressed by Mathieu functions (the time factor $\exp (-\mathrm{i} \omega t)$ is omitted here and thereafter):

$$
\begin{gathered}
u=2 \sum_{m=0}^{\infty} \mathrm{i}^{m} c e_{m}(\eta, q) M c_{m}^{(1)}(\xi, q) c e_{m}(\theta, q)+ \\
2 \sum_{m=1}^{\infty} \mathrm{i}^{m} s e_{m}(\eta, q) M s_{m}^{(1)}(\xi, q) s e_{m}(\theta, q) .
\end{gathered}
$$

More details on theory of elliptical coordinates and Mathieu functions are available in literatures (McLachlan, 1951; Morse and Feshback, 1953; Pao and Mow, 1973).

\subsection{Oblate hill}

The half-space is defined by $y \leq 0$ for an oblate hill as shown in Figure 1. The model is divided into two parts: an interior region $\Omega$ and an exterior region $D$.
$\Omega$ region is a full ellipse with the hill as its upper part by boundary $\bar{L}$ and a portion of the half-space as its lower part by boundary $\underline{L}$. $D$ region is the rest of halfspace after subtracting the full ellipse. The contact between the two regions is assumed to be welded well. Boundary $\Gamma$ is the flat surface of half-space, namely $\Gamma=\left\{(\xi, \eta) \mid \xi \in\left(\xi_{0},+\infty\right), \eta=0, \pi\right\}$. If we use $u^{\Omega}$ and $u^{D}$ to denote wave functions in regions $\Omega$ and $D$, the boundary conditions for the oblate hill are:

1) Traction-free condition at the flat surface of halfspace

$$
\left.\sigma_{\eta z}\right|_{\Gamma}=\left.\frac{\mu}{e J} \frac{\partial u^{D}}{\partial \eta}\right|_{\Gamma}=0 \quad(\xi, \eta) \in \Gamma
$$

2) Traction-free condition at the hill surface

$$
\left.\sigma_{\xi z}\right|_{\bar{L}}=\left.\frac{\mu}{e J} \frac{\partial u^{\Omega}}{\partial \xi}\right|_{\bar{L}}=0 \quad(\xi, \eta) \in \bar{L}
$$

3) Continuity of displacement and traction along the contact of two regions

$$
\left.u^{D}\right|_{\underline{L}}=\left.u^{\Omega}\right|_{\underline{L}} \text { and }\left.\quad \frac{\mu}{e J} \frac{\partial u^{D}}{\partial \xi}\right|_{\underline{L}}=\left.\frac{\mu}{e J} \frac{\partial u^{\Omega}}{\partial \xi}\right|_{\underline{L}}
$$

$$
(\xi, \eta) \in \underline{L}
$$

where $J$ is a constant and $J^{2}=(\cosh 2 \xi-\cos 2 \eta) / 2$.

The free-field solution in the absence of the hill is obtained by adding equation (9) for angle $\theta$ (incident wave) to another equation (9) for angle $-\theta$ (reflected wave). Since $\operatorname{se}_{m}(\theta, q)$ is odd in respect of $\theta$, the freefield solution is

$$
u^{\mathrm{in}+\mathrm{re}}=4 \sum_{m=0}^{\infty} \mathrm{i}^{m} c e_{m}(\eta, q) M c_{m}^{(1)}(\xi, q) c e_{m}(\theta, q) .
$$

The existence of the hill will introduce scattering waves $u^{\mathrm{S}}$ in region $D$, so the total motion in this region is $u^{D}=u^{\mathrm{in}+\mathrm{re}}+u^{\mathrm{s}}$. Since $u^{\mathrm{s}}$ and $u^{\Omega}$ must satisfy equation (3), they may be taken the following form:

$$
\begin{gathered}
u^{\mathrm{s}}=\sum_{m=0}^{\infty} a_{m} c e_{m}(\eta, q) M c_{m}^{(3)}(\xi, q)+ \\
\sum_{m=1}^{\infty} d_{m} s e_{m}(\eta, q) M s_{m}^{(3)}(\xi, q) \\
u^{\Omega}=\sum_{m=0}^{\infty} b_{m} c e_{m}(\eta, q) M c_{m}^{(1)}(\xi, q)+ \\
\sum_{m=1}^{\infty} c_{m} s e_{m}(\eta, q) M s_{m}^{(1)}(\xi, q)
\end{gathered}
$$

where $a_{m}, b_{m}, c_{m}$ and $d_{m}$ are all unknowns. Radial Mathieu functions $M c^{(3)}$ and $M s^{(3)}$ are chosen for $u^{\mathrm{s}}$ 
because they correspond to diverging wave if combined with time factor $\exp (-\mathrm{i} \omega t)$. Since $\mathrm{d} s e_{m}(\eta, q) / \mathrm{d} \eta \neq 0$ at $\Gamma$, the unknowns $d_{m}$ in equation (13) must be equal to
0 . Then, substitute $u^{\text {in+re }}$ in equation (11) and $u^{\mathrm{s}}$ in equation (12) with that in equations (10b) and (10c), yielding

$$
\begin{aligned}
& \overline{\mathbf{L}}: \sum_{m=0}^{\infty} b_{m} c e_{m}(\eta, q) M c_{m}^{(1)}\left(\xi_{0}, q\right)+\sum_{m=1}^{\infty} c_{m} s e_{m}(\eta, q) M s_{m}^{(1)}\left(\xi_{0}, q\right)=0 \\
& \underline{\mathbf{L}}: \sum_{m=0}^{\infty}\left[4 \mathrm{i}^{m} c e_{m}(\eta, q) M c_{m}^{(1)}\left(\xi_{0}, q\right) c e_{m}(\theta, q)+a_{m} c e_{m}(\eta, q) M c_{m}^{(3)}\left(\xi_{0}, q\right)\right]= \\
& \sum_{m=0}^{\infty} b_{m} c e_{m}(\eta, q) M c_{m}^{(1)}\left(\xi_{0}, q\right)+\sum_{m=1}^{\infty} c_{m} s e_{m}(\eta, q) M s_{m}^{(1)}\left(\xi_{0}, q\right) \\
& \underline{\mathbf{L}}: \mu \sum_{m=0}^{\infty}\left[4 \mathrm{i}^{m} c e_{m}(\eta, q) M c_{m}^{(1)^{\prime}}\left(\xi_{0}, q\right) c e_{m}(\theta, q)+a_{m} c e_{m}(\eta, q) M c_{m}^{(3)^{\prime}}\left(\xi_{0}, q\right)\right]= \\
& \mu \sum_{m=0}^{\infty} b_{m} c e_{m}(\eta, q) M c_{m}^{(1)^{\prime}}\left(\xi_{0}, q\right)+\mu \sum_{m=1}^{\infty} c_{m} s e_{m}(\eta, q) M s_{m}^{(1)^{\prime}}\left(\xi_{0}, q\right)
\end{aligned}
$$

Equation (5) reveals that Mathieu functions $s e_{m}(\eta, q)$ and $c e_{m}(\eta, q)$, although orthogonal in the range $[-\pi, \pi]$, are non-orthogonal in $[-\pi, 0]$ or $[0, \pi]$. So equation (14) cannot be solved directly and the non- orthogonal terms must be replaced by the orthogonal ones. Cosine series $\{\cos n \eta, n \in$ integer $\}$ satisfy this requirement, and a sine function, after even extension, can be expanded as (Lee et al., 2006)

$$
\sin r \eta=\left\{\begin{array}{lc}
-\frac{2 r}{\pi} \sum_{n=0}^{\infty} \frac{\varepsilon_{n}}{r^{2}-n^{2}} \cos n \eta & -\pi \leq \eta \leq 0 \\
\frac{2 r}{\pi} \sum_{n=0}^{\infty} \frac{\varepsilon_{n}}{r^{2}-n^{2}} \cos n \eta & 0 \leq \eta \leq \pi
\end{array} \quad n, r \in \text { integer, } n+r \in\right. \text { odd }
$$

where $\varepsilon_{0}=1$ and $\varepsilon_{n}=2$ for $n \geq 1$. Introducing equations (5) and (15) into equation (14) yields three equations that contain cosine terms only. After dropping item $\cos n \eta$ and separating equations with period of $\pi$ and
$2 \pi$ for convenience, it comes the desired form in equation (16). Every integer value of $n$ decides an equation because of the orthogonality of the series $\{\cos n \eta$, $n \in$ integer $\}$.

$$
\left\{\begin{array}{l}
\sum_{m=0}^{\infty}\left\{b_{2 m} M c_{2 m}^{(1)}\left(\xi_{0}, q\right) A_{2 n}^{2 m}-c_{2 m+1} M s_{2 m+1}^{(1)}\left(\xi_{0}, q\right) \sum_{r=0}^{\infty} \frac{B_{2 r+1}^{2 m+1}}{\pi} \frac{2(2 r+1) \varepsilon_{2 n}}{(2 r+1)^{2}-4 n^{2}}-a_{2 m} M c_{2 m}^{(3)}\left(\xi_{0}, q\right) A_{2 n}^{2 m}\right\}= \\
\quad 4 \sum_{m=0}^{\infty}(-1)^{m} c e_{2 m}(\theta, q) M c_{2 m}^{(1)}\left(\xi_{0}, q\right) A_{2 n}^{2 m} \\
\sum_{m=0}^{\infty}\left\{b_{2 m} M c_{2 m}^{(1)^{\prime}}\left(\xi_{0}, q\right) A_{2 n}^{2 m}-c_{2 m+1} M s_{2 m+1}^{(1)^{\prime}}\left(\xi_{0}, q\right) \sum_{r=0}^{\infty} \frac{B_{2 r+1}^{2 m+1}}{\pi} \frac{2(2 r+1) \varepsilon_{2 n}}{(2 r+1)^{2}-4 n^{2}}-a_{2 m} M c_{2 m}^{(3)^{\prime}}\left(\xi_{0}, q\right) A_{2 n}^{2 m}\right\}= \\
4 \sum_{m=0}^{\infty}(-1)^{m} c e_{2 m}(\theta, q) M c_{2 m}^{(1)^{\prime}}\left(\xi_{0}, q\right) A_{2 n}^{2 m} \\
\sum_{m=0}^{\infty}\left\{b_{2 m} M c_{2 m}^{(1)^{\prime}}\left(\xi_{0}, q\right) A_{2 n}^{2 m}+c_{2 m+1} M s_{2 m+1}^{(1)^{\prime}}\left(\xi_{0}, q\right) \sum_{r=0}^{\infty} \frac{B_{2 r+1}^{2 m+1}}{\pi} \frac{2(2 r+1) \varepsilon_{2 n}}{(2 r+1)^{2}-4 n^{2}}\right\}=0
\end{array}\right.
$$




$$
\left\{\begin{array}{c}
\sum_{m=0}^{\infty}\left\{b_{2 m+1} M c_{2 m+1}^{(1)}\left(\xi_{0}, q\right) A_{2 n+1}^{2 m+1}-c_{2 m+2} M s_{2 m+2}^{(1)}\left(\xi_{0}, q\right) \sum_{r=0}^{\infty} \frac{B_{2 r+2}^{2 m+2}}{\pi} \frac{8(r+1)}{4(r+1)^{2}-(2 n+1)^{2}}-\right. \\
\left.a_{2 m+1} M c_{2 m+1}^{(3)}\left(\xi_{0}, q\right) A_{2 n+1}^{2 m+1}\right\}=4 \sum_{m=0}^{\infty}(-1)^{m} \mathrm{i} c e_{2 m+1}(\theta, q) M c_{2 m+1}^{(1)}\left(\xi_{0}, q\right) A_{2 n+1}^{2 m+1} \\
\sum_{m=0}^{\infty}\left\{b_{2 m+1} M c_{2 m+1}^{(1)^{\prime}}\left(\xi_{0}, q\right) A_{2 n+1}^{2 m+1}-c_{2 m+2} M s_{2 m+2}^{(1)^{\prime}}\left(\xi_{0}, q\right) \sum_{r=0}^{\infty} \frac{B_{2 r+2}^{2 m+2}}{\pi} \frac{8(r+1)}{4(r+1)^{2}-(2 n+1)^{2}}-\right. \\
\sum_{m=0}^{\infty}\left\{b_{2 m+1} M c_{2 m+1}^{(3)^{\prime}}\left(\xi_{0}, q\right) A_{2 n+1}^{2 m+1}\right\} \\
\left.b_{m+1} M c_{2 m+1}^{(1)^{\prime}}\left(\xi_{0}, q\right) A_{2 n+1}^{2 m+1}+c_{2 m+2} M s_{2 m+2}^{(1)^{\prime}}\left(\xi_{0}, q\right) \sum_{r=0}^{\infty} \frac{B_{2 r+2}^{2 m+2}}{\pi} \frac{8(r+1)}{4(r+1)^{2}-(2 n+1)^{2}}\right\}=0
\end{array}\right.
$$

More details of the derivation can be found in $\mathrm{Fu}$ (2008). The above infinite equations can be solved after being truncated, and once all the unknowns are obtained, surface motion near the hill can be calculated.

\subsection{Prolate hill}

While for a prolate hill, it is convenient to redefine the half-space by $x \geq 0$, with the surface of the hill and the half-space represented by the thick dashed line in Figure 1. The boundaries change to

$$
\begin{aligned}
& \overline{\mathbf{L}}=\left\{(\xi, \eta) \mid \eta \in\left[-\frac{\pi}{2},-\pi\right] \bigcup\left[\frac{\pi}{2}, \pi\right), \xi=\xi_{0}\right\} \\
& \underline{\mathbf{L}}=\left\{(\xi, \eta) \mid \eta \in\left[-\frac{\pi}{2}, \frac{\pi}{2}\right], \xi=\xi_{0}\right\} \\
& \mathbf{\Gamma}=\left\{(\xi, \eta) \mid \xi \in\left(\xi_{0},+\infty\right), \eta= \pm \frac{\pi}{2}\right\}
\end{aligned}
$$

while the formula in equation (10) remain the same.

The free-field solution $u^{\text {in }+ \text { re }}$ for the prolate hill is obtained by adding equation (9) for $\theta$ (reflected wave) and equation (9) for $\pi-\theta$ (incident wave). Since $c e_{2 m+1}(\pi-\theta, q)=-c e_{2 m+1}(-\theta, q)$ and $\operatorname{se}_{2 m}(\pi-\theta, q)=$ $-s e_{2 m}(-\theta, q)$, the free-field solution becomes

$$
\begin{gathered}
u^{\mathrm{in}+\mathrm{re}}=4 \sum_{m=0}^{\infty}(-1)^{m} c e_{2 m}(\eta, q) M c_{2 m}^{(1)}(\xi, q) c e_{2 m}(\theta, q)+ \\
4 \sum_{m=0}^{\infty}(-1)^{m} \mathrm{ise}_{2 m+1}(\eta, q) M s_{2 m+1}^{(1)}(\xi, q) s e_{2 m+1}(\theta, q) .
\end{gathered}
$$

In order to satisfy equation (10a), let the scattering waves $u^{\mathrm{s}}$ be

$$
\begin{aligned}
& u^{\mathrm{s}}=\sum_{m=0}^{\infty} a_{2 m} c e_{2 m}(\eta, q) M c_{2 m}^{(3)}(\xi, q)+ \\
& \sum_{m=0}^{\infty} d_{2 m+1} s e_{2 m+1}(\eta, q) M s_{2 m+1}^{(3)}(\xi, q) .
\end{aligned}
$$

Mathieu functions $c e_{2 m}(\eta, q)$ and $s e_{2 m+1}(\eta, q)$ are orthogonal in range $[-\pi / 2,-\pi] \cup[\pi / 2, \pi)$ and $[-\pi / 2, \pi / 2]$, but $c e_{2 m+1}(\eta, q)$ and $\operatorname{se}_{2 m}(\eta, q)$ are not, so they should be treated. Setting $\eta^{\prime}=\eta+\pi / 2$ for equation (15), it comes

$$
\begin{aligned}
& \sin 2 r \eta^{\prime}= \begin{cases}-\frac{4 r}{\pi} \sum_{n=0}^{\infty} \frac{\varepsilon_{2 n+1}}{4 r^{2}-(2 n+1)^{2}}(-1)^{n-r+1} \sin (2 n+1) \eta^{\prime} & \eta^{\prime} \in\left[-\frac{\pi}{2},-\pi\right] \bigcup\left[\frac{\pi}{2}, \pi\right) \\
\frac{4 r}{\pi} \sum_{n=0}^{\infty} \frac{\varepsilon_{2 n+1}}{4 r^{2}-(2 n+1)^{2}}(-1)^{n-r+1} \sin (2 n+1) \eta^{\prime} & \eta^{\prime} \in\left[-\frac{\pi}{2}, \frac{\pi}{2}\right]\end{cases} \\
& \cos (2 r+1) \eta^{\prime}= \begin{cases}-\frac{2(2 r+1)}{\pi} \sum_{n=0}^{\infty} \frac{\varepsilon_{2 n}}{(2 r+1)^{2}-4 n^{2}}(-1)^{n-r} \cos 2 n \eta^{\prime} & \eta^{\prime} \in\left[-\frac{\pi}{2},-\pi\right] \bigcup\left[\frac{\pi}{2}, \pi\right) \\
\frac{2(2 r+1)}{\pi} \sum_{n=0}^{\infty} \frac{\varepsilon_{2 n}}{(2 r+1)^{2}-4 n^{2}}(-1)^{n-r} \cos 2 n \eta^{\prime} & \eta^{\prime} \in\left[-\frac{\pi}{2}, \frac{\pi}{2}\right]\end{cases} \\
& n, r \in \text { integer . }
\end{aligned}
$$


Then introducing equations (5) and (20) into a set of equations whose structure are similar to equation
(14), we obtain the final equation (21) for the prolate hill, and $a_{m}, b_{m}, c_{m}$ and $d_{m}$ are the unknowns.

$$
\begin{aligned}
& \int \sum_{m=0}^{\infty}\left\{b_{2 m} M c_{2 m}^{(1)}\left(\xi_{0}, q\right) A_{2 n}^{2 m}+b_{2 m+1} M c_{2 m+1}^{(1)}\left(\xi_{0}, q\right) \sum_{r=0}^{\infty}\left[\frac{(-1)^{n-r} A_{2 r+1}^{2 m+1}}{\pi} \frac{2(2 r+1) \varepsilon_{2 n}}{(2 r+1)^{2}-4 n^{2}}\right]-\right. \\
& \left.a_{2 m} M c_{2 m}^{(3)}\left(\xi_{0}, q\right) A_{2 n}^{2 m}\right\}=4 \sum_{m=0}^{\infty}(-1)^{m} c e_{2 m}(\theta, q) M c_{2 m}^{(1)}\left(\xi_{0}, q\right) A_{2 n}^{2 m} \\
& \left\{\sum _ { m = 0 } ^ { \infty } \left\{b_{2 m} M c_{2 m}^{(1)^{\prime}}\left(\xi_{0}, q\right) A_{2 n}^{2 m}+b_{2 m+1} M c_{2 m+1}^{(1)^{\prime}}\left(\xi_{0}, q\right) \sum_{r=0}^{\infty}\left[\frac{(-1)^{n-r} A_{2 r+1}^{2 m+1}}{\pi} \frac{2(2 r+1) \varepsilon_{2 n}}{(2 r+1)^{2}-4 n^{2}}\right]-\right.\right. \\
& \left.a_{2 m} M c_{2 m}^{(3)^{\prime}}\left(\xi_{0}, q\right) A_{2 n}^{2 m}\right\}=4 \sum_{m=0}^{\infty}(-1)^{m} c e_{2 m}(\theta, q) M c_{2 m}^{(1)^{\prime}}\left(\xi_{0}, q\right) A_{2 n}^{2 m} \\
& \left(\sum_{m=0}^{\infty}\left\{b_{2 m} M c_{2 m}^{(1)^{\prime}}\left(\xi_{0}, q\right) A_{2 n}^{2 m}-b_{2 m+1} M c_{2 m+1}^{(1)^{\prime}}\left(\xi_{0}, q\right) \sum_{r=0}^{\infty}\left[\frac{(-1)^{n-r} A_{2 r+1}^{2 m+1}}{\pi} \frac{2(2 r+1) \varepsilon_{2 n}}{(2 r+1)^{2}-4 n^{2}}\right]\right\}=0\right. \\
& \left\{\begin{array}{c}
\sum_{m=0}^{\infty}\left\{c_{2 m+1} M s_{2 m+1}^{(1)}\left(\xi_{0}, q\right) B_{2 n+1}^{2 m+1}+c_{2 m+2} M s_{2 m+2}^{(1)}\left(\xi_{0}, q\right) \sum_{r=0}^{\infty}\left[\frac{B_{2 r+2}^{2 m+2}}{\pi} \frac{8(-1)^{n-r}(r+1)}{4(r+1)^{2}-(2 n+1)^{2}}\right]-\right. \\
\left.d_{2 m+1} M s_{2 m+1}^{(3)}\left(\xi_{0}, q\right) B_{2 n+1}^{2 m+1}\right\}=4 \sum_{m=0}^{\infty}(-1)^{m} \mathrm{i} s e_{2 m+1}(\theta, q) M s_{2 m+1}^{(1)}\left(\xi_{0}, q\right) B_{2 n+1}^{2 m+1} \\
\sum_{m=0}^{\infty}\left\{c_{2 m+1} M s_{2 m+1}^{(1)^{\prime}}\left(\xi_{0}, q\right) B_{2 n+1}^{2 m+1}+c_{2 m+2} M s_{2 m+2}^{(1)^{\prime}}\left(\xi_{0}, q\right) \sum_{r=0}^{\infty}\left[\frac{B_{2 r+2}^{2 m+2}}{\pi} \frac{8(-1)^{n-r}(k+1)}{4(r+1)^{2}-(2 n+1)^{2}}\right]-\right. \\
\left.d_{2 m+1} M s_{2 m+1}^{(3)^{\prime}}\left(\xi_{0}, q\right) B_{2 n+1}^{2 m+1}\right\}=4 \sum_{m=0}^{\infty}(-1)^{m} \mathrm{i} s e_{2 m+1}(\theta, q) M s_{2 m+1}^{(1)^{\prime}}\left(\xi_{0}, q\right) B_{2 n+1}^{2 m+1} \\
\sum_{m=0}^{\infty}\left\{c_{2 m+1} M s_{2 m+1}^{(1)^{\prime}}\left(\xi_{0}, q\right) B_{2 n+1}^{2 m+1}-c_{2 m+2} M s_{2 m+2}^{(1)^{\prime}}\left(\xi_{0}, q\right) \sum_{r=0}^{\infty}\left[\frac{B_{2 r+2}^{2 m+2}}{\pi} \frac{8(-1)^{n-r}(k+1)}{4(r+1)^{2}-(2 n+1)^{2}}\right]\right\}
\end{array}\right.
\end{aligned}
$$

\section{Accuracy of the solutions}

Accuracy of the solution can be checked by the satisfaction extent of the boundary conditions. For this purpose, dimensionless displacement residual error $u_{b}$ and stress residual error $\sigma_{b}$ are defined as

$$
u_{b}=\frac{\left|u^{\mathrm{in}+\mathrm{re}}+u^{\mathrm{s}}-u^{\Omega}\right|}{u_{0}}
$$

and

$$
\sigma_{b}=\frac{\left|\sigma^{\mathrm{in}+\mathrm{re}}+\sigma^{\mathrm{s}}-\sigma^{\Omega}\right|}{\sigma_{0}} .
$$

The value of $\sigma_{b}$ along boundary $\bar{L}$ equals that along $\underline{L}$. Here, $u_{0}$ is the displacement amplitude of incident wave with $\sigma_{0}=-\left|\mu u_{0} \omega\right| / \beta$ representing stress amplitude of incident wave. It is convenient to define dimensionless incident frequency $\mathrm{ETA}=2 A / \lambda$ for an oblate hill and $\mathrm{ETA}=2 B / \lambda$ for a prolate hill, which describes the relationship between characteristic dimension of the hill and the incident wavelength $\lambda$. And Figures 4 and 5 illustrate the displacement and stress residual errors $u_{b}$ and $\sigma_{b}$ under the vertical incident waves $\left(\theta=90^{\circ}\right)$ for the two oblate hills and four prolate hills shown in Figure 2 . 

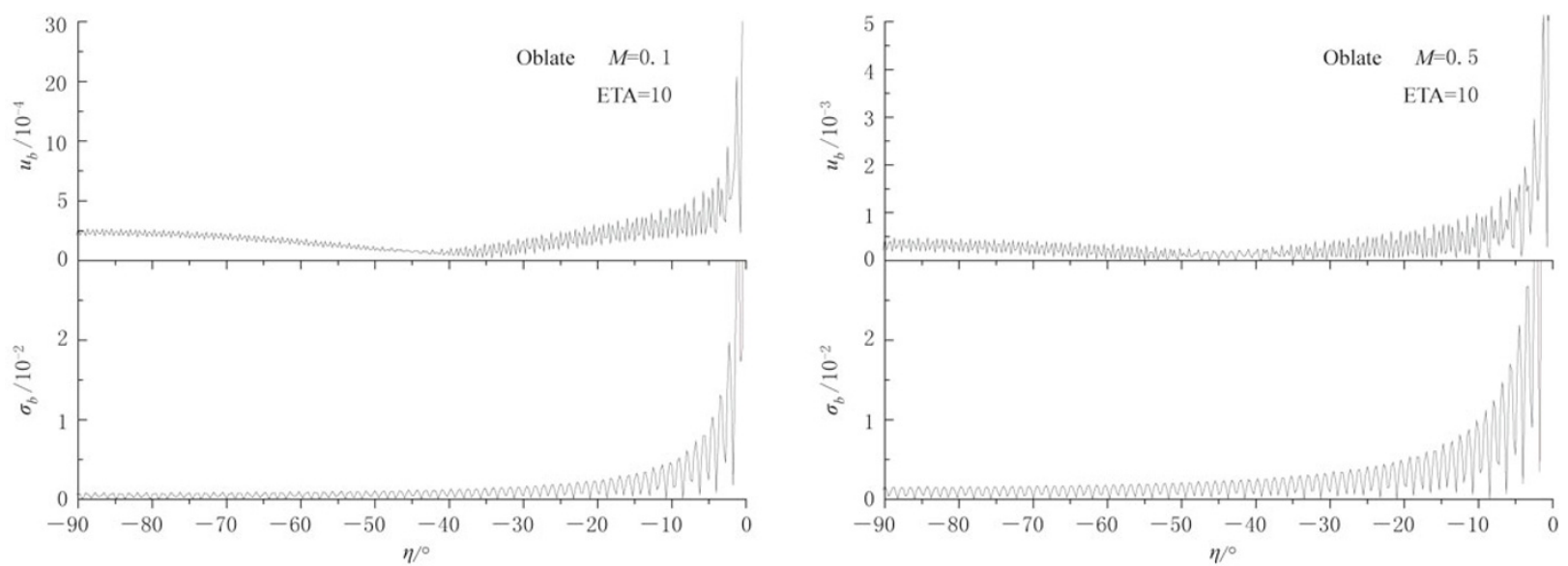

Figure 4 Displacement residual error $\left(u_{b}\right)$ and stress residual error $\left(\sigma_{b}\right)$ for oblate elliptical hill.
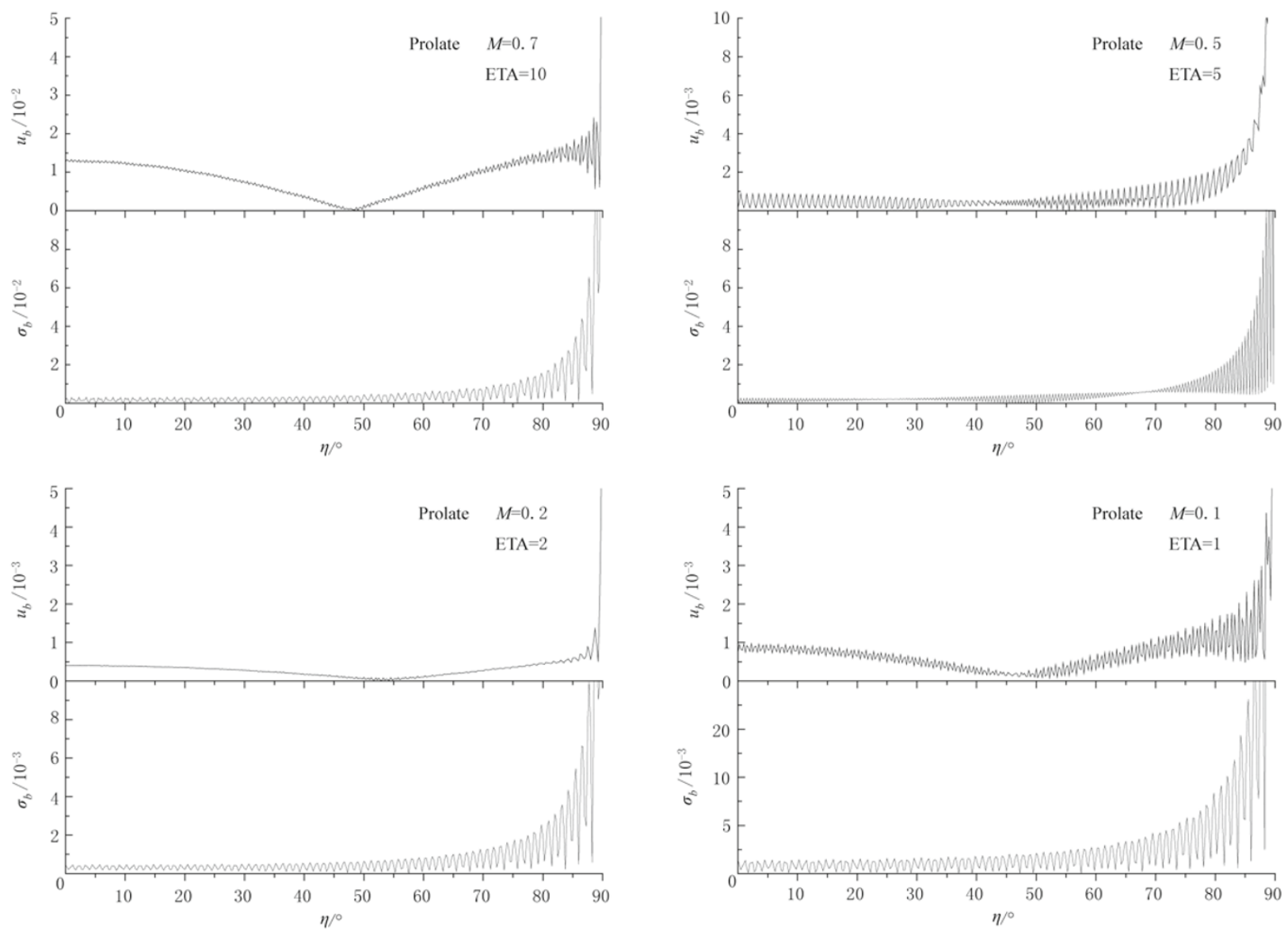

Figure 5 Displacement and stress residual error for prolate elliptical hill.

Figure 4 shows the residual error for oblate hills with $M=0.1$ and 0.5 , respectively. Because of the symmetry, only half is illustrated. It is noticed from the figures that the residual errors $u_{b}$ and $\sigma_{b}$ are relatively large near the right $\operatorname{rim}\left(\eta=0^{\circ}\right)$, and that the stress residual error $\sigma_{b}$ is much larger than the displacement residual error $u_{b}$. And for oblate hills, if $\mathrm{ETA} \leq 10$, truncation term $m=n=200$ is enough to achieve the accuracy of $10^{-3}$ for the displacement residual error $u_{b}$, except at two rims. 
The relationship between ETA, $M$ and $q$ can be written as

$$
\begin{gathered}
q=\frac{(\mathrm{ETA} \times \pi)^{2}}{4}\left(1-M^{2}\right) \quad \text { for oblate hill, } \\
q=\frac{(\mathrm{ETA} \times \pi)^{2}}{4}\left(\frac{1}{M^{2}}-1\right) \quad \text { for prolate hill. }
\end{gathered}
$$

If ETA and $M$ are beyond some values, or $q$ is very large, Mathieu functions calculation subroutines (e.g., Blanch, 1966; Leeb, 1979; Shirts, 1993a, b; Alhargan, 2000a, b, 2006) available now are still not accurate enough, and consequently, residual error may become large. According to our experience, $q \leq 180$ is roughly the limitation. Although $q>180$ does not necessarily indicate the failure of Mathieu functions, it is strongly recommended that $u_{b}$ and $\sigma_{b}$ should be checked carefully. For oblate hills, the accurate results can be obtained even when ETA is greater than 10. But for prolate hill, $q$ is easy to reach high value since $M$ in equation (23b) appears as the denominator, ETA can not be that high. Figure 5 shows the displacement and stress residual errors for some prolate hills, and ETA can be 5.0 for $M=0.5$, can be 2.0 for $M=0.2$, and can be 1.0 for $M=0.1$. However, it should be pointed out that here, once the accurate Mathieu functions for $q>180$ are available in the future, the numerical results for higher ETA can be easily achieved.

Figure 6 illustrates the displacement and stress residual errors of an oblate hill with $M=0.99$ for $\mathrm{ETA}=50$. Here $M=0.99$ represents a nearly semicircular hill, and we use $m=n=300$ for truncation. It is noticed that although ETA is extremely high here, the residual error is still very small. This is because

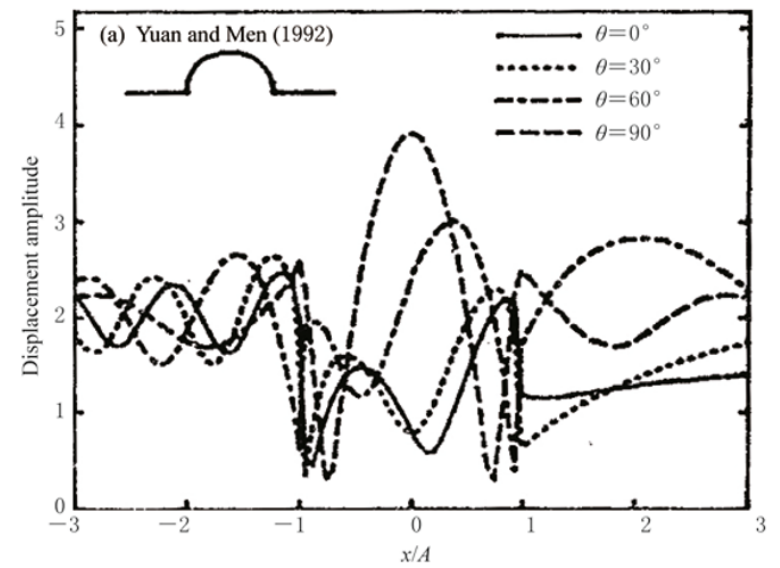

that when minor-to-major axial ratio $M \rightarrow 1$, the elliptical hill degenerates into a semi-circular hill with Mathieu functions and radial Mathieu functions reducing to trigonometric functions and Bessel functions, respectively, whose accurate values are available even under extremely high ETA. It is shown that the method in this paper is effective for an extensive range of inciden$\mathrm{t}$ frequency as long as Mathieu functions are precise enough.

Figure 7 shows the comparison of our result of nearly semi-circular hill $(M=0.99)$ for incident frequency $\mathrm{ETA}=1.0$ with that of semi-circular hill by Yuan and Men (1992). It can be seen that they agree very well with each other.

Figure 8 illustrates the comparison of our result of nearly semi-circular hill $(M=0.99)$ for incident frequency $\mathrm{ETA}=5.0$ with those of semi-circular hill by Lee et al. (2006) and Liang and $\mathrm{Ba}$ (2008). It is observed that there is nearly no difference among them.

Figures 9 and 10 show the comparison between

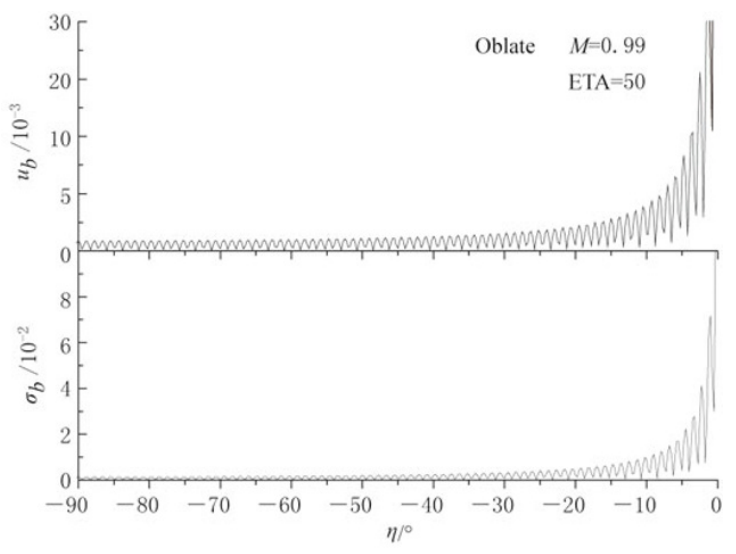

Figure 6 Displacement and stress residual error for nearly semi-circular hill.

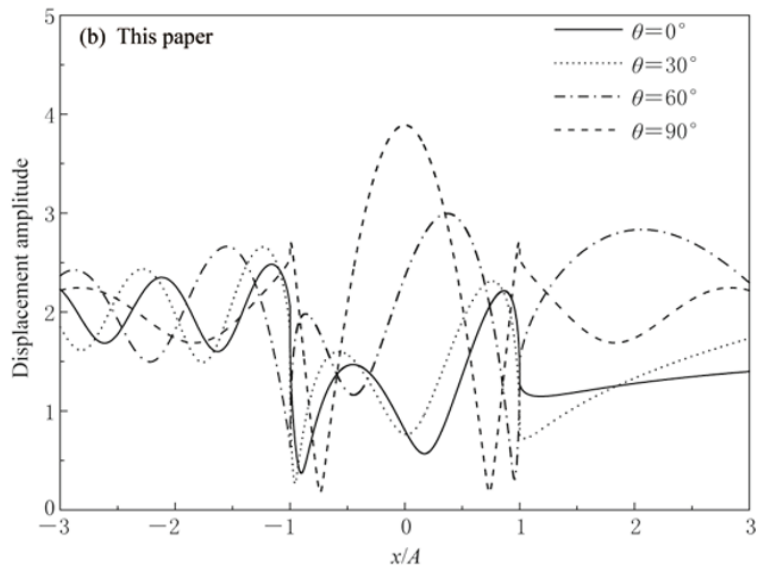

Figure 7 Comparison between the results by Yuan and Men (1992) for semi-circular hill and the results by this paper for nearly semi-circular hill with $M=0.99$ under incident frequency ETA=1.0. 


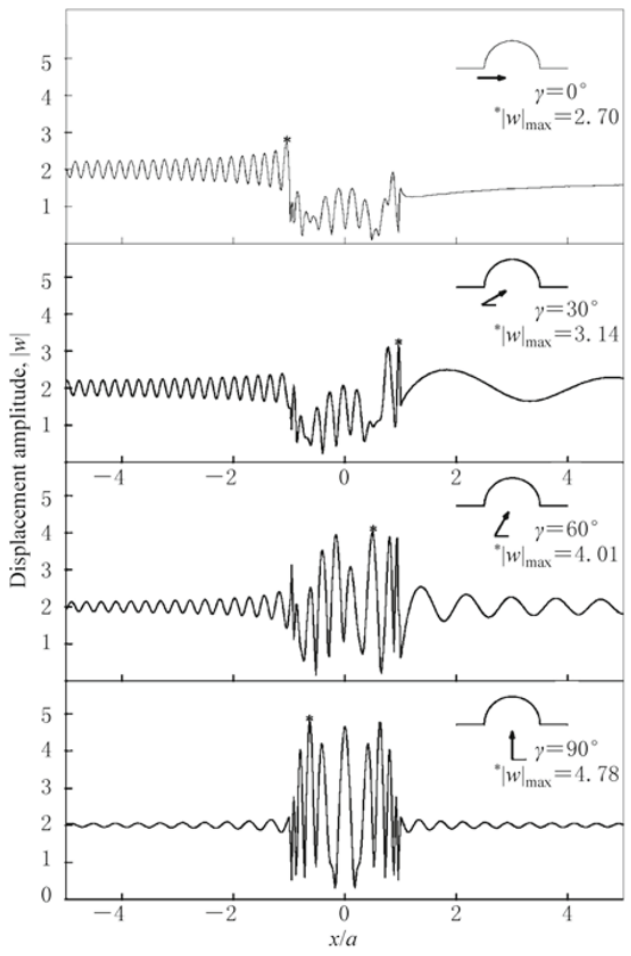

(a)

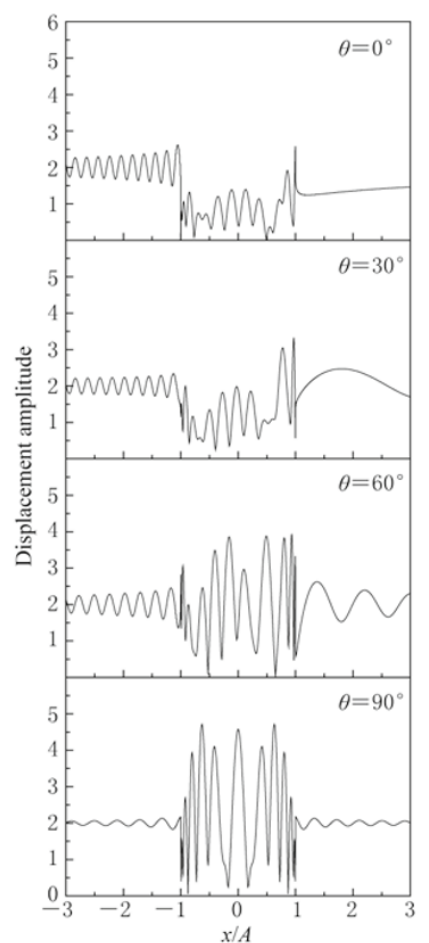

(b)

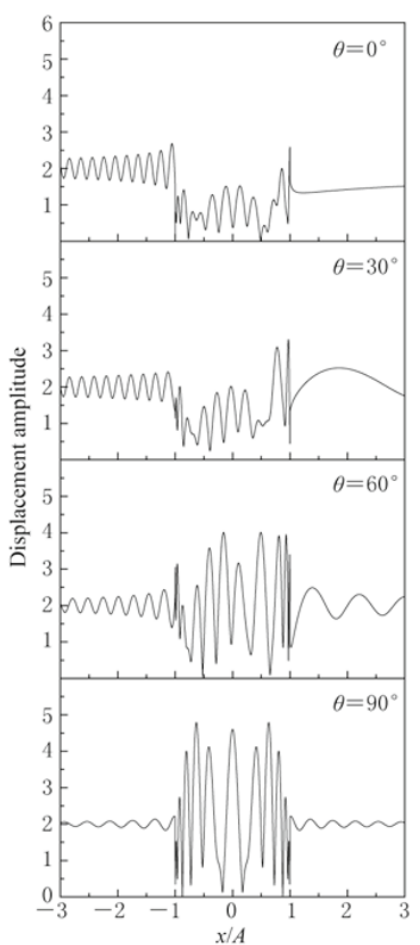

(c)

Figure 8 Comparison among the results by Lee et al. (2006) (a), by Liang and Ba (2008) (b) for semi-circular hill, and the results by this paper (c) for nearly semi-circular hill with $M=0.99$ under incident frequency ETA=5.0.
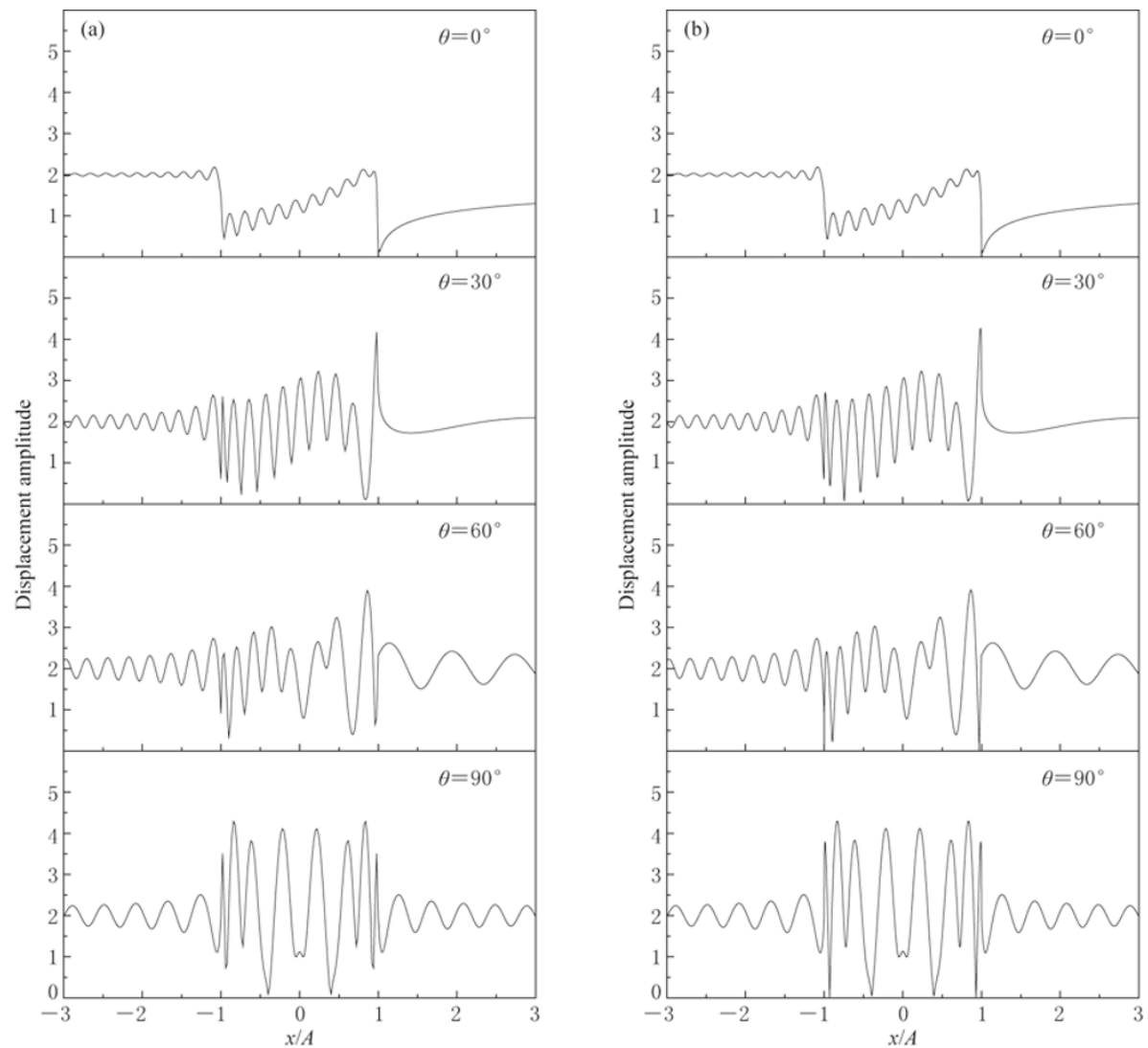

Figure 9 Comparison between the results by Lee and Amornwongpaibum (2011) (a) and by this paper (b) for oblate hill with $M=0.5$ under incident frequency $\mathrm{ETA}=5.0$. 

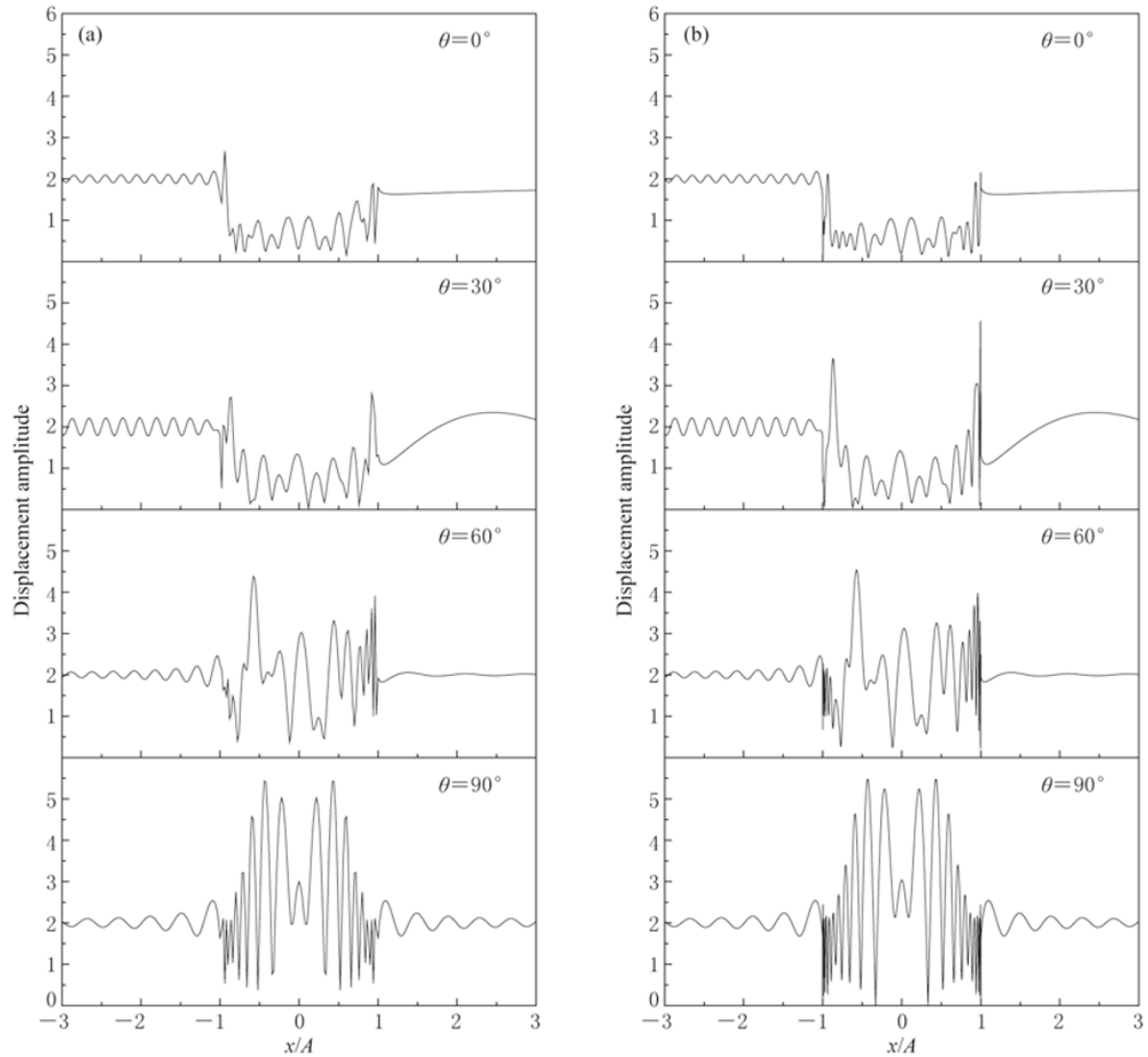

Figure 10 Comparison between results by Amornwongpaibum and Lee (2011) (a) and by this paper (b) for prolate hill with $M=1 / 1.9$ under incident frequency $\mathrm{ETA}=5.0$.

the results by Lee and Amornwongpaibum (2011) and by this paper for oblate hill with $M=0.5$ under incident frequency $\mathrm{ETA}=5.0$, and the comparison between the results by Amornwongpaibum and Lee (2011) and by this paper for prolate hill with $M=1 / 1.9$ under incident frequency $\mathrm{ETA}=5.0$. It can be seen that both comparisons are in good agreement.

Here, it should be pointed out that, this paper, Lee and Amornwongaibun (2011) and Amornwongaibun and Lee (2011) are finished independently, with different objective and different way of solution. To match the coefficients of elliptic sine and cosine terms, this paper expands elliptic cosine in terms of trigonometric cosine, and elliptic sine in terms of trigonometric sine, as in Wong and Trifunac (1974b), then uses the orthogonality of trigonometric cosine to express trigonometric sine in terms of trigonometric cosine. This approach is more classical. On the other hand, Lee and Amornwongaibun (2011) and Amornwongaibun and Lee (2011) expressed elliptic sine directly in terms of elliptic cosine, saving an intermediate step of trigonometric sine and cosine. This paper aims at very prolate hill, with $M$ being taken up to 0.1 , while $M$ is only taken up to $1 / 1.9$ in Amornwon- gaibun and Lee (2011).

\section{Numerical results and analysis}

The displacement amplitude and its phase can be defined as

$$
\text { amplitude }=|u|
$$

and

$$
\text { phase }=\arctan [\operatorname{Im} u / \operatorname{Re} u] / \pi .
$$

In the absence of local topography, surface displacement amplitude equals 2 everywhere and its phase would be a straight line

$$
\begin{aligned}
\operatorname{phase}(u)= & -\frac{\omega}{\pi \beta} x=-\frac{\omega A}{\pi \beta} \frac{x}{A} \cos \theta= \\
& -\frac{x}{A} \mathrm{ETA} \cos \theta .
\end{aligned}
$$

As the hill introduces scattering waves, the amplitude and phase deviate from their original shapes.

Figures 11 through 16 illustrate surface motion of the oblate and prolate hills shown in Figure 2. The upper part of each sub-figure represents displacement 

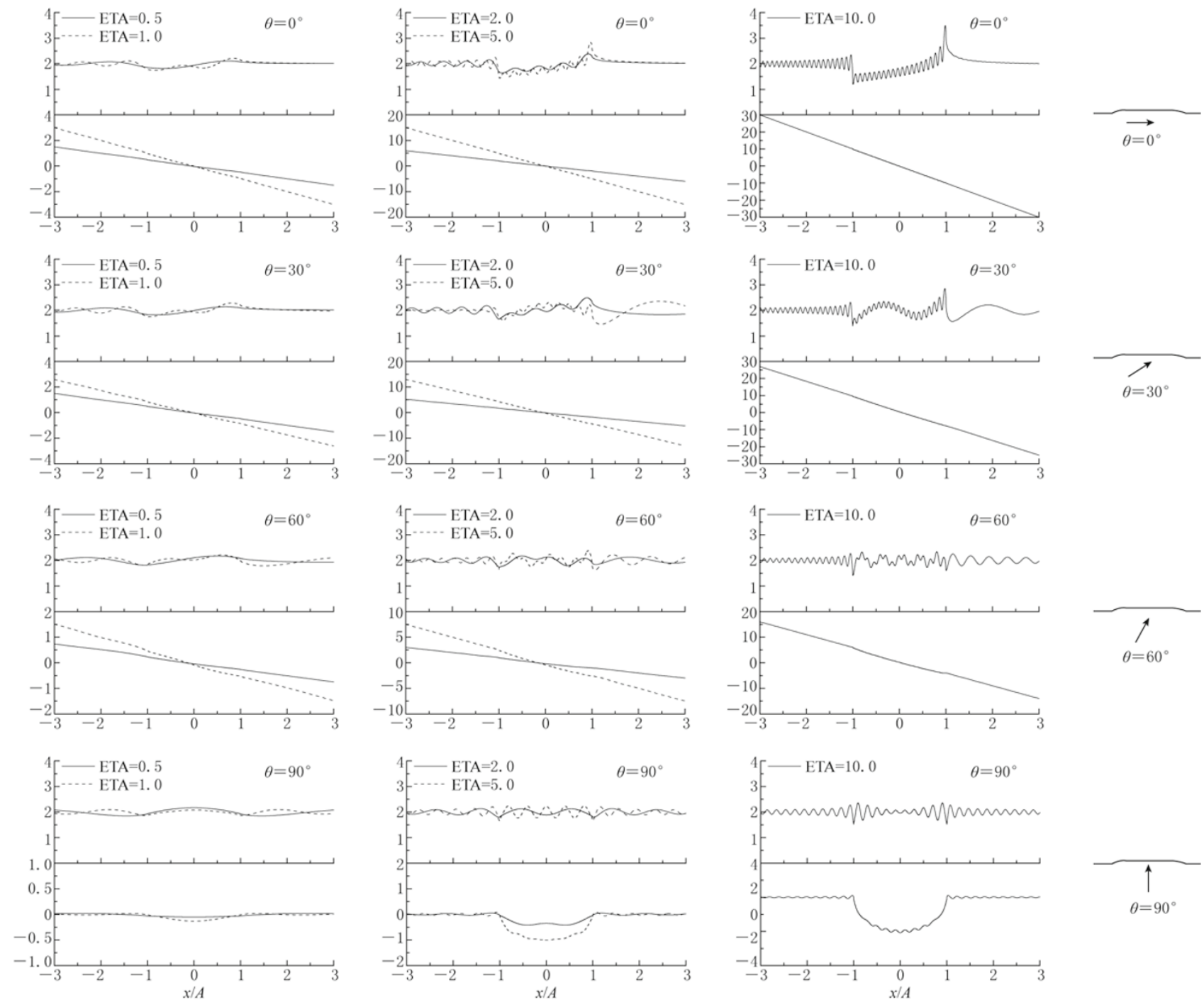

Figure 11 Surface displacement amplitude and phase for oblate elliptical hill $(M=0.1)$. In each sub-figure, the upper part illustrates surface displacement amplitudes, with the lower part relevant phase.

amplitude with the lower representing its phase. Horizontal axis in Figures 11 and 12 is $x / A$ for oblate hills, and horizontal axis in Figures 13 to 16 is $y / B$ for prolate hills.

Figures 11 and 12 show surface motion of oblate hill with minor-to-major axial ratios $M=0.1$ and $M=0.5$, respectively, with incident frequency $\mathrm{ETA}=0.5$, 1.0, 2.0, 5.0, and 10.0, and with incident angle $\theta=0^{\circ}$, $30^{\circ}, 60^{\circ}$ and $90^{\circ}$. For $M=0.1$, the hill is very oblate and just likes a step. When ETA is low, the displacement amplitude fluctuates mildly around 2 and its phase is roughly a straight line. As ETA increases, the displacement amplitude gradually vibrates wildly, and its peak become larger especially for $\mathrm{ETA}=10$. While for $M=0.5$, the surface displacement amplitude increases signifi- cantly, and its phase becomes more complex. In region $-1 \leq x / A \leq 1$ (hill surface), the displacement amplitudes are larger and fluctuate more vigorously for high incident frequencies, also the nearly-standing waves can be observed here. With incident angle $\theta$ increasing, the displacement amplitude on hill surface increases correspondingly, and with ETA increasing, the displacement amplitude on the hill surface increases in general. Surface displacement amplitude in two sides $x / A<-1$ and $x / A>1$ is less complicated. For vertical incidence of waves, the displacement amplitude is much larger on the hill surface than the half-space surface, while for grazing incidence of waves, shadow zone is evident on and behind the hill. 

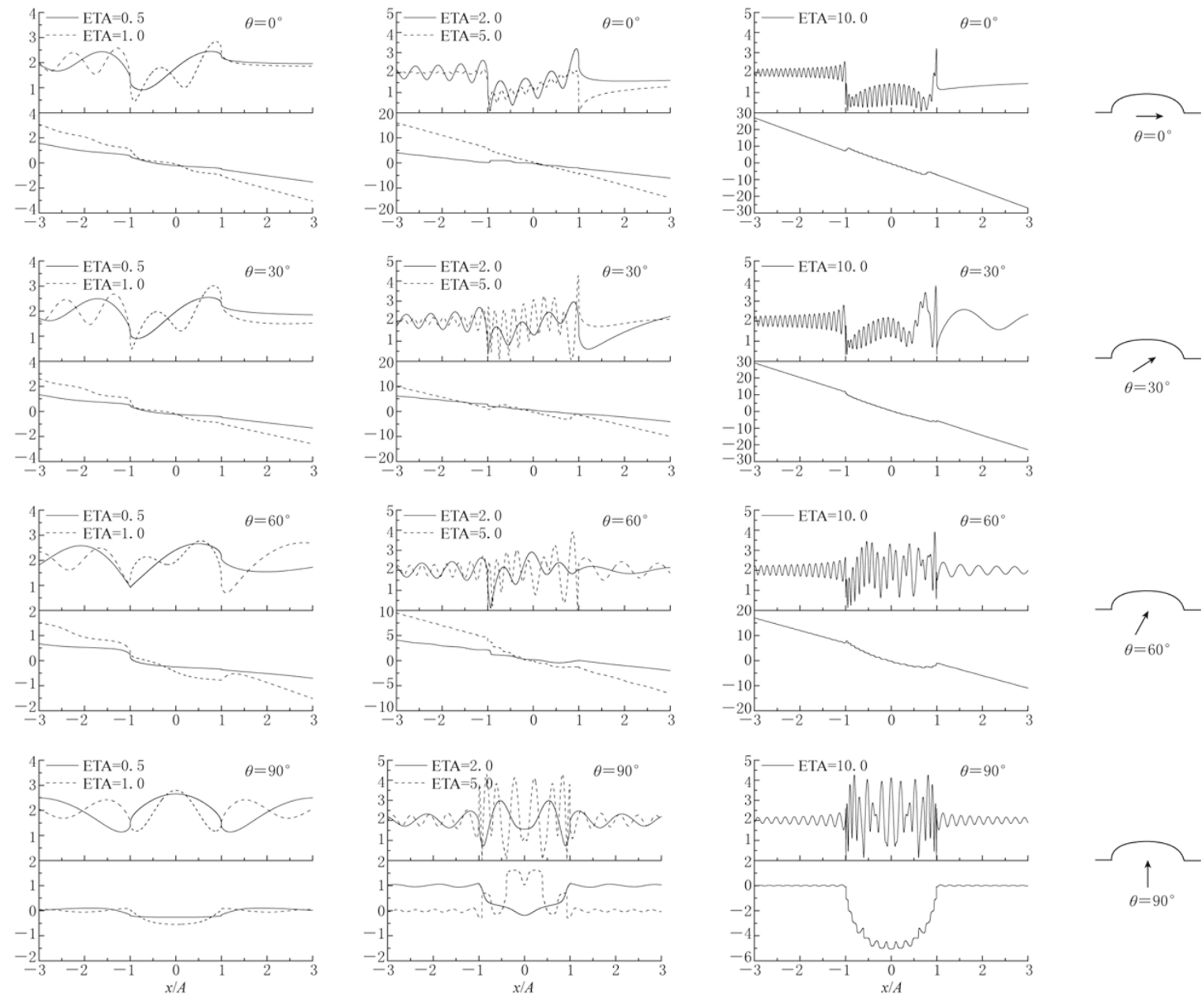

Figure 12 Surface displacement amplitude and phase for oblate elliptical hill $(M=0.5)$. In each sub-figure, the upper part illustrates surface displacement amplitude, with the lower part relevant phase.

Figures 13 through 16 show the surface motion for prolate hills with the minor-to-major axial ratios $M=0.7,0.5,0.2$ and 0.1 , respectively, with incident angle $\theta=0^{\circ}, 30^{\circ}, 60^{\circ}$ and $90^{\circ}$, and with incident frequency ETA having different limit for different $M$. In order to be consistent with Figures 11 and 12 for oblate hills, $\theta=0^{\circ}$ still corresponds to grazing incidence of waves and $\theta=90^{\circ}$ to vertical incidence of waves. It is shown that with ETA increasing, the displacement amplitude vibrates more vigorously and the displacement amplitude increases for large incident angle $\left(\theta=90^{\circ}\right.$ or $\left.\theta=60^{\circ}\right)$. For the given ETA, the displacement amplitude on the hill surface is larger for more prolate (i.e., with smaller $M$ ) ones because the waves are reflected forth-and-back inside the hill more probably, which is a special property in prolate hill. While for the given $M$, the displacement amplitude on the hill surface increases with increasing $\theta$ and the displacement amplitude reaches its maximal value for $\theta=90^{\circ}$. As for very prolate hills (e.g., with $M=0.2$ and $M=0.1$ ), the extremely standard standing waves with essential $180^{\circ}$ phase change can be easily observed on the hill surface, with the phase roughly being a straight line on the half-space surface. This is also a special phenomenon in prolate hill.

\section{Conclusions}

This paper presents a closed-form analytical solution of surface motion of a semi-elliptical cylindrical hill for incident plane SH waves using the sine and cosine 

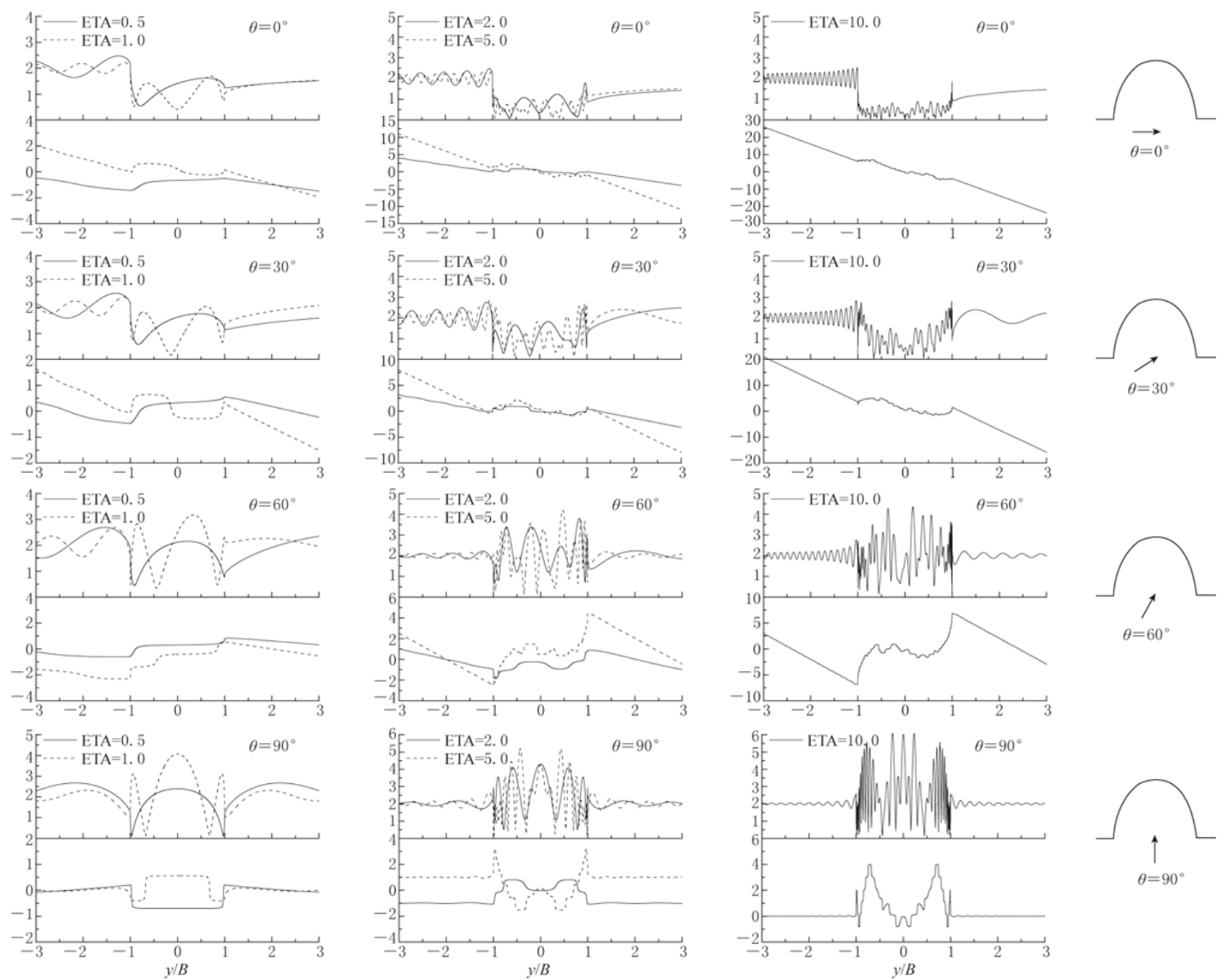

Figure 13 Surface displacement amplitude and phase for prolate elliptical hill $(M=0.7)$. In each sub-figure, the upper part illustrates surface displacement amplitudes, with the lower part relevant phase.
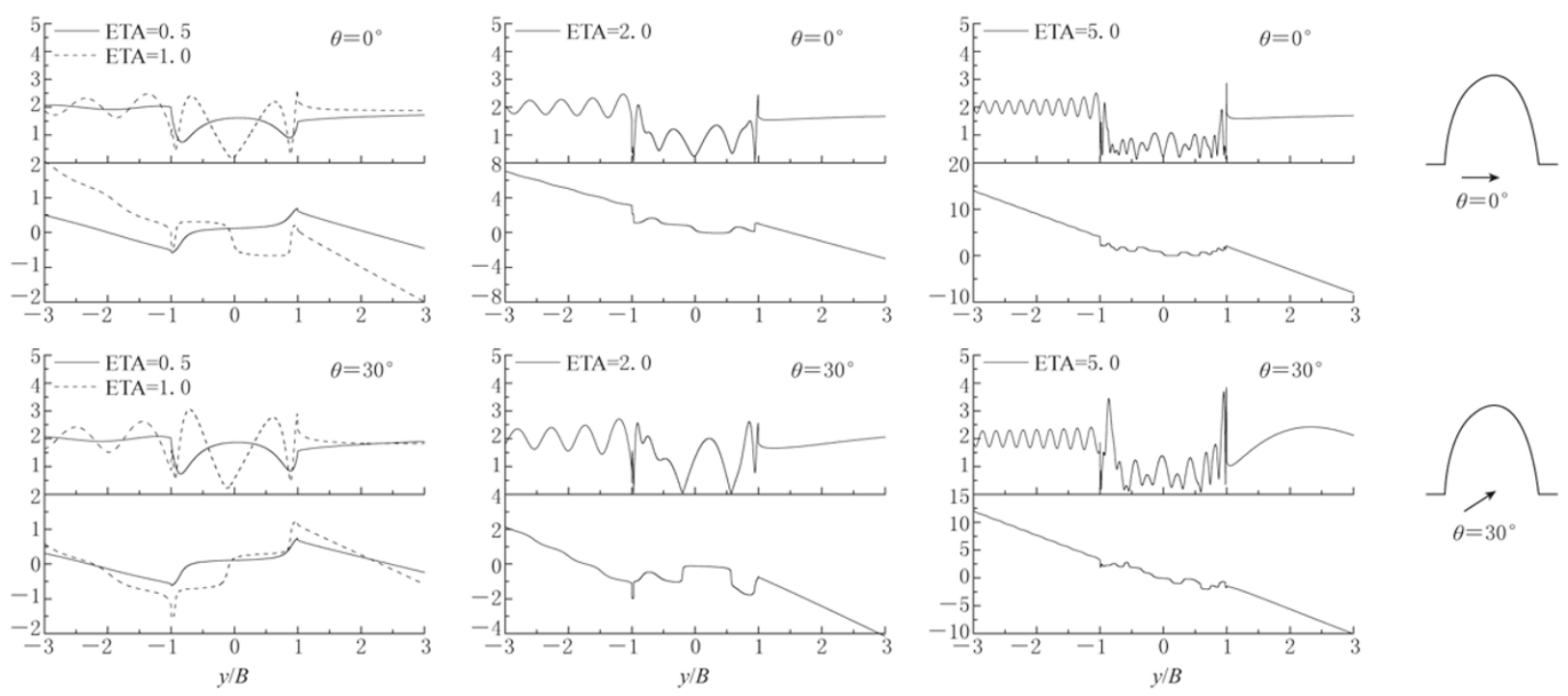

Figure 14 Surface displacement amplitude and phase for prolate elliptical hill $(M=0.5)$. In each sub-figure, the upper part illustrates surface displacement amplitude, with the lower part relevant phase. 

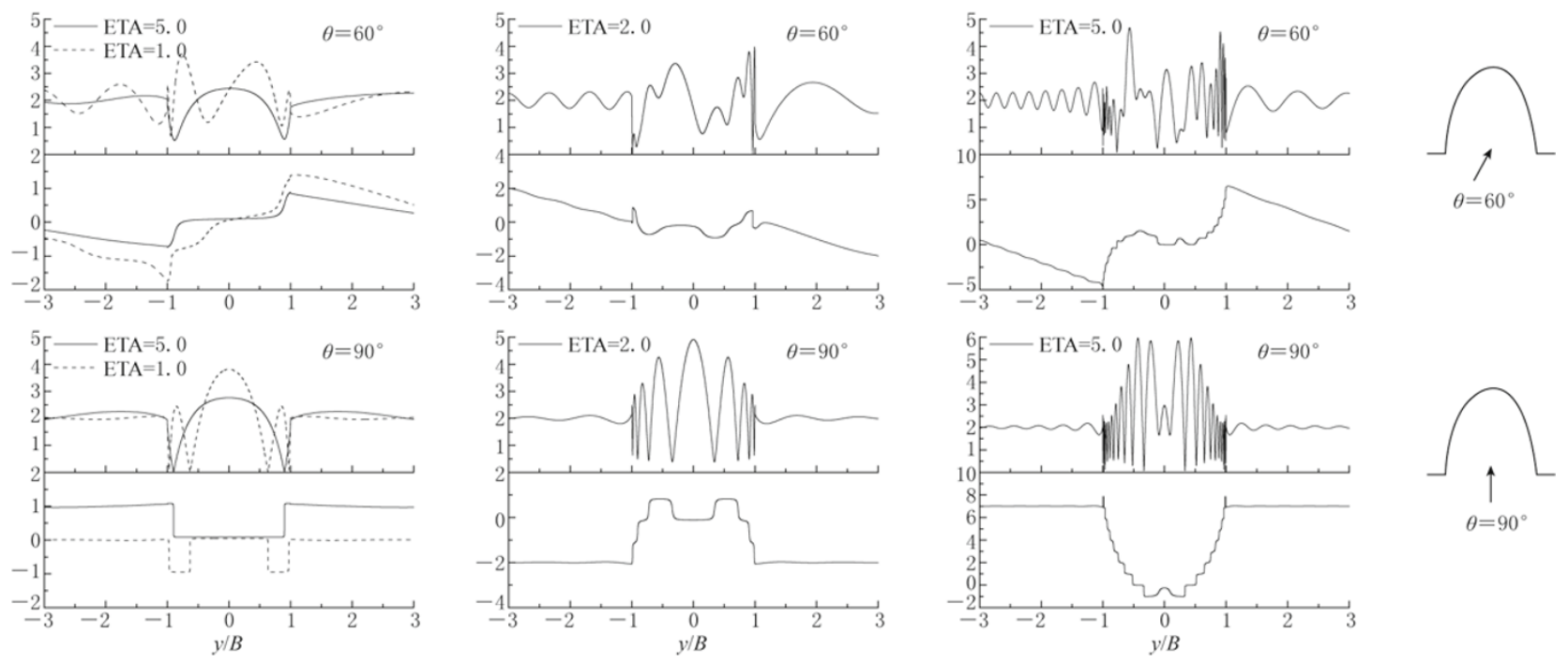

Figure 14 Surface displacement amplitude and phase for prolate elliptical hill $(M=0.5)$. In each sub-figure, the upper part illustrates surface displacement amplitude, with the lower part relevant phase.
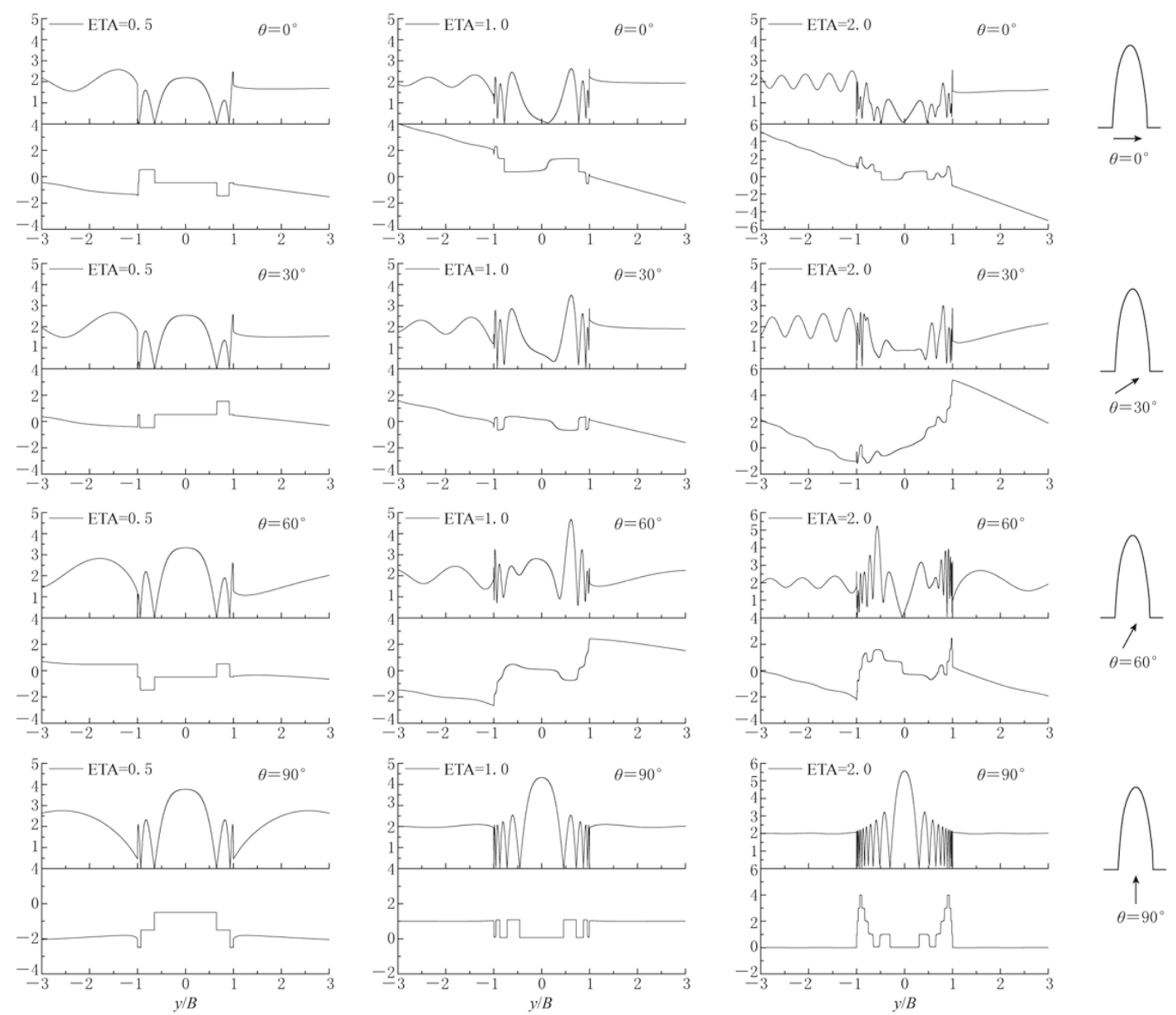

Figure 15 Surface displacement amplitude and phase for prolate elliptical hill $(M=0.2)$. In each sub-figure, the upper part illustrates surface displacement amplitude, with the lower part relevant phase. 

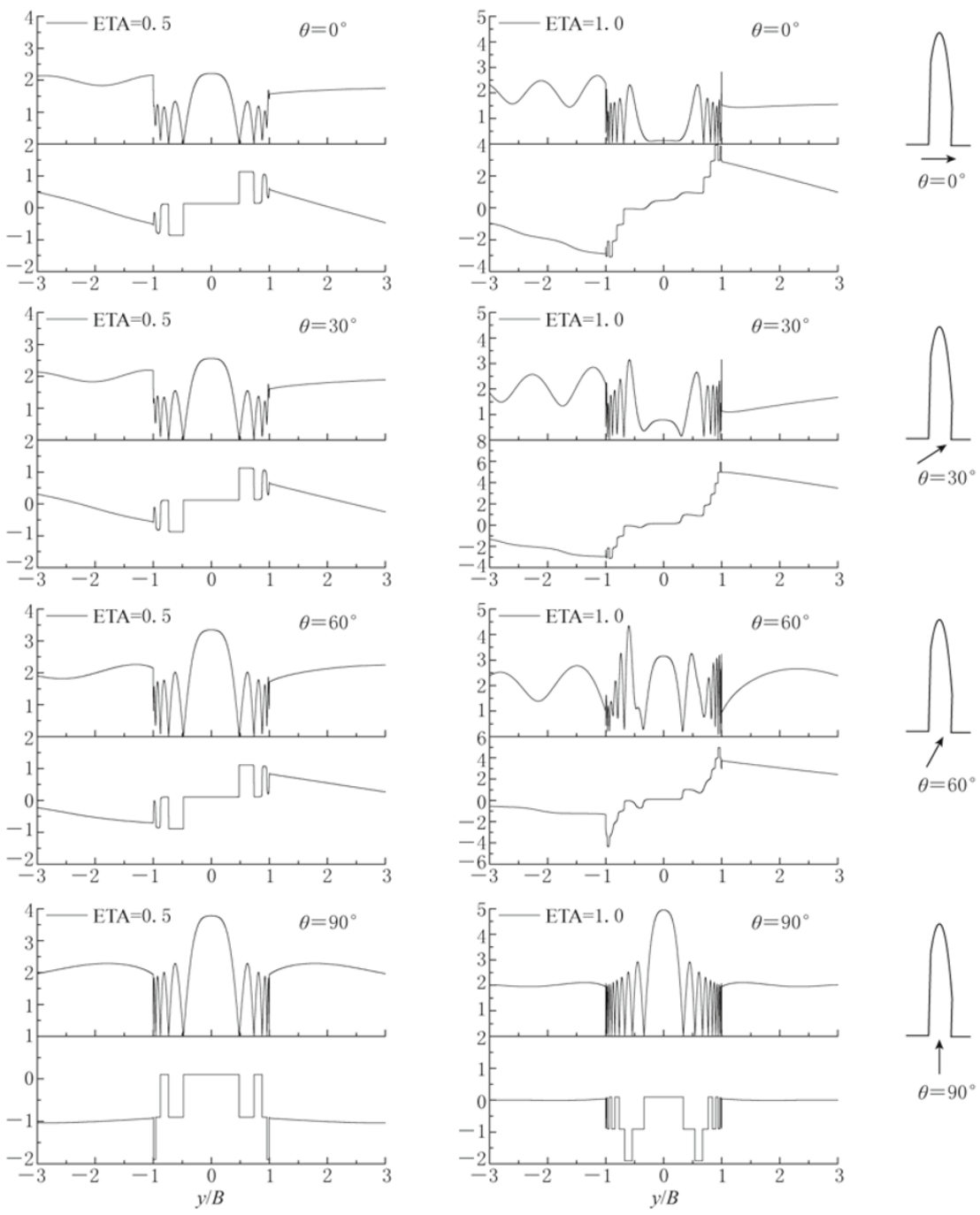

Figure 16 Surface displacement amplitude and phase for prolate elliptical hill $(M=0.1)$. In each sub-figure, the upper part illustrates surface displacement amplitude, with the lower part relevant phase.

series expansion technique, and accuracy of the solution is checked by the boundary conditions. Numerical results for surface motion of oblate and prolate hills are calculated, and some conclusions can be obtained.

In terms of subroutines of Mathieu functions available now, incident frequency can be very high for oblate hills; while for very prolate hills, incident frequency can not be that high. However, excellent numerical results have been obtained for some very prolate hills in this paper. If more accurate Mathieu functions subroutines are available in the future, this problem can be solved.

Surface motion around a hill depends significantly on hill shape, incident frequency, and incident angle, and may fluctuate rapidly over short distances. For vertical incidence of waves, the displacement amplitude is much larger on hill surface than on half-space, while for grazing incidence of waves, shadow zone is more evident for more prolate hill. For the given incident frequency, the displacement amplitude on the hill surface is larger for more prolate hill, because waves are reflected forthand-back inside the hill more probably. For very prolate hill, the extremely standard standing waves can be easily observed even for low incident frequency.

Acknowledgements This study is supported by National Natural Science Foundation of China under grant No. 50978183, which is gratefully acknowledged.

\section{References}

Alhargan F A (2000a). Algorithms for the computation of all Mathieu functions of integer orders. ACM Trans Math Software 26: 390-407.

Alhargan F A (2000b). Algorithms 804: Subroutines for 
the computation of Mathieu functions of integer orders. ACM Trans Math Software 26: 408-414.

Alhargan F A (2006). Algorithm 855: Subroutines for the computation of Mathieu characteristic numbers and their general orders. ACM Trans Math Software 32: 472484 .

Amornwongaibun A and Lee V W (2011). Scattering of antiplane (SH) waves by a semi-elliptical hill: II — deep hill. Soil Dynamics and Earthquake Engineering (in review).

Blanch G (1966). Numerical aspects of Mathieu eigenvalues. Rend Circ Mat Paler 15: 51-97.

Fu J (2008). Scattering of Plane SH Wave by a Semielliptical Hill. [Master Dissertation]. Department of Civil Engineering, Tianjin University, Tianjin, 7-23 (in Chinese with English abstract)

Lee V W and Amornwongaibun A (2011). Scattering of antiplane $(\mathrm{SH})$ waves by a semi-elliptical hill: I - shallow hill. Soil Dynamics and Earthquake Engineering (in review).

Lee V W, Luo H and Liang J (2006). Antiplane (SH) waves diffraction by a semicircular cylindrical hill revisited: An improved analytical wave series solution. J Eng Mech, ASCE 132: 1 106-1 113.

Leeb W (1979). Algorithm 537: Characteristic values of Mathieu's differential equation. ACM Trans Math Software 5: 112-117.

Liang J and Ba Z N (2008). Surface motion of a hill in layered half-space subjected to incident plane $\mathrm{SH}$ waves. Earthq Eng Eng Vibr 28: 1-10.

McLachlan N W (1951). Theory and Application of Mathieu Functions. Clarendon Press, Oxford, 259pp.

Morse P L and Feshback H (1953). Methods of Theoretical Physics. McGraw-Hill Book Company, New York, 1 4071432 .
Pao Y H and Mow C C (1973). Diffraction of Elastic Waves and Dynamics Stress Concentration. Crane, Russak \& Company Inc., New York, 401-450.

Shirts R B (1993a). The computation of eigenvalues and solutions of Mathieu's differential equation for noninteger order. ACM Trans Math Software 19: 377-390.

Shirts R B (1993b). Algorithm 721 MTIEU1 and MTIEU2: Two subroutines to compute eigenvalues and solutions to Mathieu's differential equation for noninteger and integer order. ACM Trans Math Software 19: 391-406.

Trifunac M D (1971). Surface motion of a semi-cylindrical alluvial valley for incident plane $\mathrm{SH}$ waves. Bull Seismol Soc Am 61: 1 755-1 770.

Trifunac M D (1973). Scattering of plane SH waves by a semi-cylindrical canyon. Earthq Eng Struct Dyn 1: 267281.

Wong H L and Trifunac M D (1974a). Scattering of plane SH wave by a semi-elliptical canyon. Earthq Eng Struct Dyn 3: 157-169.

Wong H L and Trifunac M D (1974b). Surface motion of a semi-elliptical alluvial valley for incident plane $\mathrm{SH}$ waves. Bull Seismol Soc Am 64: 1 389-1 408.

Yuan X M and Liao Z P (1994). Scattering of plane SH waves by a cylindrical canyon of circular-arc cross-section. Soil Dyn Earthq Eng 13: 407-412.

Yuan X M and Liao Z P(1995). Scattering of plane SH waves by a cylindrical alluvial valley of circular-arc crosssection. Earthq Eng Struct Dyn 25: 1 303-1 313.

Yuan X M and Liao Z P (1996). Surface motion of a cylindrical hill of circular-arc cross-section for incident plane SH waves. Soil Dyn Earthq Eng 15: 189-199.

Yuan X M and Men F L (1992). Scattering of plane SH waves by a semi-cylindrical hill. Earthq Eng Struct Dyn 21: 1 091-1 098. 